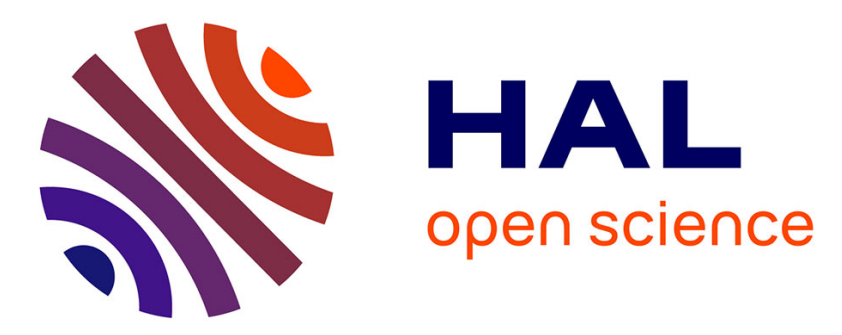

\title{
Maladies infectieuses émergentes : des processus complexes, difficiles à prédire
}

\author{
Jean-François Guégan
}

\section{To cite this version:}

Jean-François Guégan. Maladies infectieuses émergentes : des processus complexes, difficiles à prédire. Biologie Aujourd'hui, 2016, 210 (4), pp.205-209. 10.1051/jbio/2016023 . hal-02393188

\section{HAL Id: hal-02393188 \\ https://hal.umontpellier.fr/hal-02393188}

Submitted on 4 Dec 2019

HAL is a multi-disciplinary open access archive for the deposit and dissemination of scientific research documents, whether they are published or not. The documents may come from teaching and research institutions in France or abroad, or from public or private research centers.
L'archive ouverte pluridisciplinaire HAL, est destinée au dépôt et à la diffusion de documents scientifiques de niveau recherche, publiés ou non, émanant des établissements d'enseignement et de recherche français ou étrangers, des laboratoires publics ou privés. 


\title{
Maladies infectieuses émergentes : des processus complexes, difficiles à prédire
}

\author{
Jean-François Guégan \\ UMR MIVEGEC, IRD-CNRS-Université de Montpellier, Centre IRD de Montpellier, BP 64501, 34394 Montpellier \\ Cedex 5, France \\ Auteur correspondant : Jean-François Guégan, jean-francois.guegan@ird.fr
}

Reçu le $1^{\text {er }}$ juillet 2016

\begin{abstract}
Résumé - À la lumière d'une double approche, d'abord empirique, illustrée par l'exemple de l'ulcère de Buruli et de son agent mycobactérien Mycobacterium ulcerans sur lesquels j'ai concentré mon activité de recherche ces dix dernières années, puis théorique et comparative, en m'intéressant aux déterminants et facteurs d'émergence de nouveaux agents infectieux ou parasitaires, la complexité des événements en cause pour expliquer le phénomène d'émergence est présentée. Les cascades d'événements, intervenant à différents niveaux d'échelles spatio-temporelles et d'organisation du vivant, et qui conduisent aux nombreuses émergences observées, exigent aujourd'hui de mieux prendre en compte les interactions hôte(s)/pathogène(s)/environnement, en y incluant les comportements des individus et de la population. Dans de nombreux travaux de recherche sur les maladies infectieuses émergentes, il s'agit d'un danger infectieux qui est décrit et non un risque, ce dernier résultant de la confrontation entre une association de phénomènes menaçants, ou aléas, et une population susceptible. Au-delà, le sujet des maladies infectieuses émergentes et de ses liens avec les changements environnementaux et sociétaux globaux entraîne à devoir reconsidérer certaines connaissances bien établies en infectiologie et parasitologie.
\end{abstract}

Mots clés : Ulcère de Buruli / maladies infectieuses émergentes / infectiologie / parasitologie

\begin{abstract}
Emerging infectious diseases: complex, unpredictable processes.
In the light of a double approach, at first empirical, later theoretical and comparative, illustrated by the example of the Buruli ulcer and its mycobacterial agent Mycobacterium ulcerans on which I focused my research activity these last ten years by studying determinants and factors of emerging infectious or parasitic diseases, the complexity of events explaining emerging diseases will be presented. The cascade of events occurring at various levels of spatiotemporal scales and organization of life, which lead to the numerous observed emergences, nowadays requires better taking into account the interactions between host(s), pathogen(s) and the environment by including the behavior of both individuals and the population. In numerous research studies on emerging infectious diseases, microbial hazard is described rather than infectious disease risk, the latter resulting from the confrontation between an association of threatening phenomena, or hazards, and a susceptible population. Beyond, the theme of emerging infectious diseases and its links with global environmental and societal changes leads to reconsider some well-established knowledge in infectiology and parasitology.
\end{abstract}

Key words: Buruli ulcer / emerging infectious diseases / infectiology / parasitology

L'ulcère de Buruli est une des Maladies Tropicales Négligées (MTN) les moins étudiées au monde, malgré son fort pouvoir invalidant et une incidence annuelle autour de 5 à 10000 cas par an. Dans le cadre d'un programme financé par l'Agence Nationale de la Recherche (ANR EXTRA-MU) et d'une Jeune 
Équipe Associée Internationale (JEAI) de l'IRD au Cameroun d'une part, et du LabEx Centre d'Étude de la Biodiversité Amazonienne (CEBA) et de son programme transversal BIOHOPSYS sur les relations entre biodiversité et maladies infectieuses en Guyane française d'autre part, nous nous sommes intéressés à : (1) quantifier l'importance des facteurs environnementaux, abiotiques et biotiques, sur les dynamiques écologiques de Mycobacterium ulcerans, l'agent responsable de l'ulcère de Buruli, dans et entre sites endémiques et non endémiques; (2) identifier le mode de transmission de $M$. ulcerans de l'environnement aux populations humaines; (3) comprendre et interpréter le caractère saisonnier des épidémies de cas d'ulcère de Buruli, révélé pour la première fois, et relier la variabilité observée de cas au long terme avec les changements environnementaux globaux; et (4) caractériser les effets de l'ulcère de Buruli sur les inégalités économiques au sein des populations des régions endémiques du Cameroun. Dans un premier temps, nous avons réalisé un important travail sur le terrain, au Cameroun et en Guyane française, afin de décrire les dynamiques spatio-temporelles de $M$. ulcerans dans les écosystèmes aquatiques au sein de deux régions endémiques à ulcère de Buruli (Akonolinga et Bankim) au Cameroun et le long de la bande côtière habitée en Guyane française où la maladie sévit de manière occasionnelle. En parallèle sur les deux continents, nous avons collecté et classifié taxonomiquement plusieurs centaines de milliers d'organismes aquatiques ainsi que les conditions physico-chimiques de l'environnement dans 32 sites au Cameroun et 18 sites en Guyane. Ces différents sites ont été suivis de manière régulière et systémique au cours d'une année. La présence, ou l'absence, ainsi que l'abondance relative de $M$. ulcerans dans nos échantillons et par sites ont été estimées par PCR et PCR quantitative. Les résultats principaux de ce travail mettent en évidence le caractère très ubiquiste de $M$. ulcerans dans les environnements aquatiques camerounais et guyanais (Garchitorena et al., 2014; Morris et al., 2014a). Le bacille est présent dans la plupart des écosystèmes aquatiques prospectés jusque dans des sites très éloignés de toute activité humaine, il est présent chez la plupart des organismes aquatiques échantillonnés (près de 90 groupes taxonomiques différents d'invertébrés et de vertébrés sont porteurs du bacille), et il se maintient au cours du temps dans ces écosystèmes. Néanmoins, nous identifions des périodes au cours de l'année (saison des pluies) et des écosystèmes (zones marécageuses et de bas-fonds) plus favorables, ce qui suggère que les dynamiques de cette mycobactérie sont le résultat d'interactions complexes entre facteurs abiotiques et biotiques dans les environnements aquatiques où réside ce bacille (Garchitorena et al., 2015a). Les caractéristiques environnementales qui fa- vorisent la persistance et la prolifération de cette mycobactérie au Cameroun et en Guyane sont quasiidentiques, mais nous notons cependant des différences plus importantes entre les deux régions d'Akonolinga et de Bankim au Cameroun marquées pour la première par un environnement équatorial et pour la seconde par des conditions de transition vers la savane arborée sèche, qu'entre Akonolinga et les sites guyanais tous caractérisés par des conditions équatoriales. En plus de fournir l'étude présentant le plus grand jeu de données spatio-temporelles au monde sur l'écologie de $M$. ulcerans, ce travail interroge à la fois sur la problématique des échantillonnages en microbiologie et en infectiologie souvent très limités spatialement et temporellement (Carolan et al., 2014a) ainsi que sur les classifications officielles actuelles en zone endémique versus non endémique pour ce type de maladie infectieuse alors qu'en réalité l'agent microbien responsable est beaucoup plus largement distribué que dans les seuls foyers où sévit l'infection (Carolan et al., 2014b).

Combinant des techniques statistiques multivariées de type modèles linéaires généralisés avec des procédures de sélection multi-modèles, nous avons montré sur la base des données acquises au Cameroun qu'au moins deux modalités de persistance du bacille dans les écosystèmes aquatiques coexistent. Lorsque les conditions abiotiques (température, $p H$, ions,...) lui sont favorables, $M$. ulcerans peut persister librement dans l'eau et contaminer les organismes aquatiques directement, et ce selon des processus densité-dépendants. En revanche, lorsque ces conditions abiotiques sont défavorables, $M$. ulcerans se maintient dans les écosystèmes aquatiques au sein des réseaux trophiques notamment par les relations proies-prédateurs (Garchitorena et al., 2015a). Des travaux de biologie expérimentale menés dans notre équipe montrent que des concentrations en chitine et des valeurs de $p H$ différentes conditionnent la croissance de la mycobactérie avec un préférentiel de $p H$ modifié autorisant le développement du bacille pour des valeurs plus acides lorsque l'environnement est riche en chitine (Sanhueza et al., 2016). Ces résultats importants correspondent certainement à une biologie complexe de ce bacille et à l'existence de plusieurs phénotypes de $M$. ulcerans qui restent encore à explorer. En Guyane française, sur la vingtaine de sites surveillés, nous avons pu reconstruire à l'aide de mesures d'isotopes stables sur les échantillons à notre disposition chacun des réseaux trophiques locaux et mettre en correspondance les différents maillons de ces réseaux avec les niveaux de concentrations en mycobactéries. Les résultats révèlent que les maillons trophiques les plus bas concentrent la mycobactérie soit en la filtrant comme le font certains mollusques bivalves d'eau douce soit, encore, l'ingurgitent au cours de leurs repas 
comme le font les espèces détritivores ou brouteuses par exemple (Morris et al., 2016b). Ces résultats sont aussi confirmés par une de nos études effectuées au Ghana mettant en évidence l'existence de groupes alimentaires de certaines espèces aquatiques, essentiellement des invertébrés comme des larves d'insectes ou des mollusques, ayant un rôle essentiel dans le processus de concentration du bacille (Morris et al., 2016a). De plus, les milieux dulçaquicoles lents (marécages, plaines inondables,...) paraissent jouer un rôle important dans l'épidémiologie de la transmission de $M$. ulcerans par rapport aux habitats courants (rivières, fleuves,... ) (García-Peña et al., 2016).

Pour les mêmes sites guyanais, la mise en relation des observations épidémiologiques avec les caractéristiques environnementales de déforestation (pourcentage de déforestation dans un rayon de $5 \mathrm{~km}$ autour du site) et de modification d'usage des sols pour le développement d'une agriculture (en pourcentage dans un rayon de $5 \mathrm{~km}$ ) conduit aux observations suivantes. Les sites montrant une forte concentration en mycobactéries sont préférentiellement les sites les plus déforestés des dernières années ainsi que ceux où s'est pratiquée une modification d'habitats pour le développement d'une agriculture. À l'inverse, les sites pas ou peu déforestés révèlent des concentrations en mycobactéries par site moins importantes (Morris et al., 2016b). La déforestation apparaît comme le facteur prépondérant pour rendre compte d'une augmentation de concentration en $M$. ulcerans dans les environnements aquatiques proches. La désorganisation des communautés animales aquatiques qu'engendre la déforestation entraîne préférentiellement une disparition des organismes prédateurs, laissant alors les espèces de proie proliférer dans ces environnements. L'explosion des proies dans ces sites déforestés et exploités pour l'agriculture est liée aux organismes des groupes alimentaires décrits plus haut. Par cascade d'événements, la déforestation conduit à ce que le processus de prédation naturel ne s'opère plus ou de manière moins efficace, laissant un second processus, celui de concentration du micro-organisme, se développer (Meyin à Ebong et al., soumis). En défrichant la forêt primaire pour le développement d'une agriculture de subsistance ou plus intensive comme en Côte d'Ivoire pour la production du riz, des humains s'exposent alors à un micro-organisme environnemental, lequel, s'il pénètre au travers de l'épiderme et du derme entraîne des ulcères caractéristiques d'un feu bactérien (Landier et al., 2014).

Le mode de transmission de $M$. ulcerans des écosystèmes aquatiques - qui représentent le premier facteur de risque de la maladie - aux populations humaines est très mal compris aujourd'hui, ce qui gêne considérablement la mise en place de stratégies de prévention et de lutte efficace contre l'ulcère de Buruli.
Les deux hypothèses principales de transmission sont une transmission vectorielle par la piqûre/morsure de punaises aquatiques (ordre Hemiptera) et une transmission environnementale par contact entre la peau de l'individu - une plaie semble toutefois nécessaire - et l'environnement contaminé. Afin de quantifier la contribution de ces deux modes de transmission du bacille dans les régions d'Akonolinga et Bankim au Cameroun, nous avons combiné nos données environnementales sur $M$. ulcerans avec des séries temporelles géo-référencées de cas d'ulcère de Buruli dans des modèles épidémiologiques et statistiques. Nous avons ainsi pu montrer, pour la première fois, que la transmission de la mycobactérie à partir de l'environnement vers les populations humaines joue un rôle bien plus important dans ces régions endémiques du Cameroun que la transmission actuellement admise par un insecte vecteur (Garchitorena et al., 2015b). S'il ne rejette pas l'hypothèse d'une transmission vectorielle cependant hautement peu probable ou peu fréquente, ce travail, publié dans le journal Nature Scientific Reports, suggère que la biologie de la transmission de la mycobactérie à l'humain est bien plus complexe qu'acceptée à l'heure actuelle, et que plusieurs scénarios de transmission peuvent exister, voire coexister, localement et dépendent des conditions environnementales décrites plus haut.

La dynamique saisonnière de la mycobactérie, $M$. ulcerans, dans les environnements aquatiques au Cameroun et en Guyane française est semblable à un bloom bactérien bénéficiant de conditions abiotiques et biotiques favorables pour proliférer. Ce phénomène intervient aux environs de mars à avril dans les deux régions prospectées. Nous avons pu relier pour la première fois cette dynamique saisonnière du bacille environnemental avec les cas d'ulcère de Buruli qui apparaissent environ 3 à 4 mois après le développement de la bactérie. L'ulcère de Buruli en Guyane et au Cameroun montre ainsi une saisonnalité dans l'apparition des cas ce qui tend à conforter l'importance de facteurs environnementaux dans l'épidémiologie de cette pathologie humaine. En Guyane, l'obtention d'une série temporelle de cas suffisamment longue (plus de 40 ans d'enregistrement) nous a aussi permis de relier l'apparition de cas d'ulcère dans la population humaine avec des changements climatiques régionaux et globaux (Morris et al., 2014b). À un niveau régional, la Guyane française est située dans une zone potentiellement à risque de relativement faible pluviométrie en lien avec l'Oscillation Australe El Niño, et dans laquelle des cas d'ulcère de Buruli peuvent potentiellement se déclarer après l'apparition d'un événement El Niño. La réduction des pluies et de leurs écoulements entraîne, en effet, la multiplication de zones d'eaux stagnantes résiduelles, où prolifère la bactérie. La plus grande accessibilité des habitats marécageux qui 
en résulte facilite leur fréquentation par les humains (pêche, chasse, etc.) et intensifie ainsi leur exposition au micro-organisme persistant dans ce type d'environnement aquatique (Morris et al., 2014b).

$\mathrm{Si}$ les incidences et prévalences pour cette maladie restent faibles, l'ulcère de Buruli contribue cependant à affecter économiquement les familles et les villages qui en sont atteints en privant les individus malades de l'accès au travail ou à l'éducation, par exemple. Combinant des données à notre disposition et des modèles épidémiologiques et économiques, nous avons pu montrer que les maladies tropicales rares mais très handicapantes comme l'ulcère de Buruli, peuvent avoir des conséquences importantes sur les groupes socioéconomiques les plus défavorisés comme au Cameroun par exemple où nous avons mené cette étude (Garchitorena et al., 2015c), et par conséquent entraîner des inégalités très importantes au niveau de la population. Les villages affectés par ce type de maladies, souvent co-occurrentes puisqu'on peut aussi y trouver de la bilharziose ou des loases, sont affaiblis économiquement et tombent dans une spirale qui les extrait du développement.

En adoptant une démarche OneHealth/EcoHealth, notre travail original montre que l'agent microbien causant l'ulcère de Buruli est beaucoup plus largement distribué que sur les sites endémiques où sévit la maladie. Il suggère plusieurs voies de transmission probables et dépendantes du contexte environnemental et humain. L'exposition de populations humaines à cet aléa microbiologique lors de l'exploitation des écosystèmes naturels pour le développement de l'agriculture en zones intertropicales entraîne les infections. Enfin, notre étude estime pour la première fois que ces MTN rares mais très handicapantes ont cependant un poids socioéconomique important sur les villages, ce qui les empêche d'accéder à un développement acceptable. Comme pour de nombreuses maladies infectieuses émergentes, de multiples paramètres et facteurs environnementaux, abiotiques et biotiques, culturels, comportementaux et socio-économiques interviennent pour rendre compte du processus d'émergence selon des cascades d'événements souvent mal compris car finalement peu étudiés, et ne correspondant pas à la vision encore très linéaire de la recherche sur les maladies infectieuses. Aujourd'hui, de nombreuses maladies infectieuses émergentes peuvent aussi s'expliquer à partir de l'exemple que nous décrivons dans cet article.

\section{Références}

Carolan K., Garchitorena A., García-Peña G.E., Morris A., Landier J., Fontanet A., Le Gall P., Texier G.,
Marsollier L., Gozlan R.E., Eyangoh S., Lo Seen D., Guégan J.-F (2014a). Topography and Land Cover of Watersheds Predicts the Distribution of the Environmental Pathogen Mycobacterium ulcerans in Aquatic Insects. PLoS Negl Trop Dis 8, e3298.

Carolan K., Meyin à Ebong S., Garchitorena A., Landier J., Sanhueza D., Texier G., Marsollier L., Le Gall P., Guégan J.-F., Lo Seen D. (2014b). Ecological niche modelling of Hemipteran insects in Cameroon; the paradox of a vector-borne transmission for Mycobacterium ulcerans, the causative agent of Buruli ulcer. Int $J$ Health Geogr 13, 44.

Garchitorena A., Roche B., Kamgang R., Ossomba J., Babonneau J., Landier J., Fontanet A., Flahault A., Eyangoh S., Guégan J.-F., Marsollier L. (2014). Mycobacterium ulcerans Ecological Dynamics and Its Association with Freshwater Ecosystems and Aquatic Communities : Results from a 12-Month Environmental Survey in Cameroon. PLoS Negl Trop Dis 8, e2879.

Garchitorena A., Guégan J.-F., Léger L., Eyangoh S., Marsollier L., Roche B. (2015a). Mycobacterium ulcerans dynamics in aquatic ecosystems are driven by a complex interplay of abiotic and biotic factors. eLife 4, 1-19.

Garchitorena A., Ngonghala C.N., Texier G., Landier J., Eyangoh S., Bonds M.H., Guégan J.-F., Roche B. (2015b). Environmental transmission of Mycobacterium ulcerans drives dynamics of Buruli ulcer in endemic regions of Cameroon. Sci Rep 5, 18055.

Garchitorena A., Ngonghala C.N., Guégan J.-F., Texier G., Bellanger M., Bonds M., Roche B. (2015c). Economic inequality caused by feedbacks between poverty and the dynamics of a rare tropical disease : the case of Buruli ulcer in sub-Saharan Africa. Proc $R$ Soc Lond Biol Sci, 282, 20151426.

García-Peña G.E., Garchitorena A., Carolan K., Canard E., Prieur-Richard A.-H., Suzan G.E., Mills J.N., Roche B., Guégan J.-F. (2016). Niche-based host extinction increases prevalence of an environmentallyacquired pathogen. Oikos, 10.1111/oik.02700.

Landier J., Gaudart J., Carolan K., Lo-Seen D., Guégan J.F., Eyangoh S., Fontanet A., Texier G. (2014). Spatiotemporal patterns and landscape-associated risk of Buruli ulcer in Akonolinga, Cameroon. PLoS Negl Trop Dis, 8, e3123

Meyin à Ebong S., García-Peña G.E., Pluot-Sigwalt D., Marsollier L., Le Gall P., Eyangoh S., Guégan J.-F. (en révision). Ecology drives infection of water bugs with Mycobacterium ulcerans in Africa. EcoHealth.

Morris A., Gozlan R.E., Marion E., Marsollier L., Andreou D., Sanhueza D., Ruffine R., Couppié P., Guégan J.F. (2014a). First detection of Mycobacterium ulcerans DNA in Environmental Samples from South America. PLoS Negl Trop Dis, 8, e2660. 
Morris A., Gozlan R.E., Hassani H., Andreou D., Couppié P., Guégan J.-F. (2014b). Complex temporal climate signals drive the emergence of human water-borne disease. Emerg Microbes Infect, 3, e56.

Morris A., Guégan J.-F., Benbow M.E., Williamson H., Small P.L.C., Quaye C., Boakye D., Merritt R.W., Gozlan R.E. (2016a). Functional diversity as a new framework for understanding the ecology of emerging generalist pathogen. EcoHealth, 13, 570-581.
Morris A., Guégan J.-F., Andreou D., Marsollier L., Carolan K., Le Croller M., Babonneau J., Sanhueza D., Gozlan R.E. (2016b). Environmentally-mediated food web collapse promotes infectious human disease carrying species in tropical freshwater systems. Science Advances (sous presse).

Sanhueza D., Chevillon C., Babonneau J., Marion E., Marsollier L., Guégan J.-F. (2016). Chitin promotes Mycobacterium ulcerans growth. FEMS Microbiol Ecol, 92, fiw067. 